Paula Matos Oliveira ${ }^{1}$

Rita Elizabeth Mascarenhas ${ }^{2}$

SUZANA RAMOS FERRER ${ }^{2}$

Rone Peterson Cerqueira Oliveira ${ }^{3}$

IANE ÉRICA MARQues TRAVESSA ${ }^{4}$

Marques Vinícius de Castro Gomes ${ }^{4}$

Maria FERNANDA RIOS GRASSI ${ }^{5}$

\section{Vulvovaginites em mulheres infectadas pelo vírus da imunodeficiência humana}

\author{
Vaginal infections in buman immunodeficiency virus-infected women
}

Palavras-chave

HIV

Vaginose bacteriana

Candidíase

Tricomoníase

Vulvovaginite

Questionários

Keywords

HIV

Vaginosis, bacterial

Candidiasis

Trichomonas infections

Vulvovaginitis

Questionnaires

\section{Resumo}

OBJETIVO: comparar a freqüência de vulvovaginites em mulheres infectadas pelo vírus da imunodeficiência humana (HIV) com mulheres não infectadas. MÉTODOS: estudo de corte transversal com 64 mulheres infectadas pelo HIV e 76 não infectadas. Foram calculadas as freqüências de vaginose bacteriana, candidíase e tricomoníase, que foram diagnosticadas por critérios de Amsel, cultura e exame a fresco, respectivamente. Para análise dos dados, utilizaram-se o teste do $\chi^{2}$, teste exato de Fisher e regressão múltipla para verificar a independência das associações. RESULTADOS: a infecção vaginal foi mais prevalente em pacientes infectadas pelo HIV quando comparadas ao Grupo Controle 159,4 versus 28,9\%, $\mathrm{p}<0,001$; Odds Ratio=2,7, IC95\%=1,33-5,83, $\mathrm{p}=0,007)$. Vaginose bacteriana ocorreu em 26,6\% das mulheres HIV positivas; candidíase vaginal, em 29,7\% e tricomoníase, em 12,5\%. Todas foram significativamente mais freqüentes no grupo de mulheres infectadas pelo HIV (p=0,04, 0,02 e 0,04, respectivamente). CONCLUSÕES: vulvovaginites são mais freqüentes em mulheres infectadas pelo HIV.

\section{Abstract}

PURPOSE: to compare the frequency of vulvovaginitis in women infected with human imunnodeficiency virus (HIV) with the frequency in non-infected women. METHODS: a transversal study including 64 HIV infected women and 76 noninfected ones. The frequencies of bacterial vaginosis, candidiasis and trichomoniasis, diagnosed by Amsel's criteria, culture and fresh exam, respectively, were calculated. Chi-square test, Fisher's exact test and multiple regressions to verify the independence of associations were used to analyze the data. RESULTS: the vaginal infection was more prevalent in HIV infected patients, as compared to the control group 159.4 versus 28.9\%, $p<0,001$; Odds Ratio=2.7, $\mathrm{IC} 95 \%=1.33-5.83, \mathrm{p}=0.007)$. Bacterial vaginosis occurred in $26.6 \%$ of the positive-HIV women; vaginal candidiasis, in $29.7 \%$ and trichomoniasis, in $12.5 \%$ of them. All the infections were significantly more frequent in the group of HIV infected women ( $p=0.04,0.02$ e 0.04 , respectively). CONCLUSIONS: vulvovaginitis is more frequent in HIV infected women.
Correspondência:

Maria Fernanda Rios Grassi Laboratório Avancado de Saúde Pública Centro de Pesquisa Gonçalo Moniz - CPaGM Rua Waldemar Falcão, 121, Candea CEP 40296-710 - Salvador/BA E-mail: grassi@bahia.fiocruz.br

Recebido

13/9/2007

Aceito com modificacões
Trabalho realizado no Curso de Pós-graduação de Medicina e Saúde Humana da Escola Bahiana de Medicina e Saúde Pública - EBMSP - Salvador (BA), Brasil. Pesquisa fomentada pela Fundação de Amparo à Pesquisa do Estado da Bahia (Fapesb) - Bahia (BA), Brasil. ' Pós-graduanda do Programa de Pós-graduação em Medicina e Saúde e Professora de Ginecologia da Escola Bahiana de Medicina e Saúde Pública - EBMSP - Salvador (BA), Brasil.

2 Doutora; Professora de Microbiologia da Escola Bahiana de Medicina e Saúde Pública- EBMSP - Salvador (BA), Brasil. ${ }^{3}$ Pós-graduando do Programa de Pós-graduação em Medicina e Saúde da Escola Bahiana de Medicina e Saúde Pública - EBMSP - Salvador (BA), Brasil.

${ }^{4}$ Acadêmico de Medicina da Escola Bahiana de Medicina e Saúde Pública - EBMSP - Salvador (BA), Brasil; Bolsista de Iniciação Científica da Fundação de Amparo a Pesquisa da Bahia - Fabesp - Bahia (BA), Brasil.

${ }_{5}^{5}$ Doutora; Professora do Programa de Pós-graduação em Medicina e Saúde da Escola Bahiana de Medicina e Saúde Pública EBMSP - Salvador (BA), Brasil; Pesquisadora Associada da Fundação Oswaldo Cruz - Fiocruz - Bahia (BA), Brasil. 


\section{Introdução}

Estima-se em 40 milhões o número de indivíduos infectados pelo vírus da imunodeficiência humana (HIV) no mundo, dos quais a metade é mulheres ${ }^{1}$. No Brasil, a prevalência da infecção pelo HIV é de até $0,5 \%$, observando-se uma tendência atual de expansão da epidemia entre as mulheres e um deslocamento dos grandes centros urbanos da região sudeste para as demais regiões geográficas. Desta forma, a razão homem/mulher entre os infectados tem sofrido uma redução gradual de 18,5 na década de 1980 para 1,5 em 2004. A maior taxa de incidência em mulheres foi observada em 2003: 14,1 casos/100 mil mulheres ${ }^{2}$.

A vulvovaginite é um dos problemas ginecológicos mais comuns, sendo o corrimento genital uma das 25 razões mais freqüentes pela qual a mulher procura atendimento médico ${ }^{3}$. A vaginose bacteriana (VB), a candidíase vaginal e a tricomoníase são responsáveis por 90\% dos casos das vulvovaginites infecciosas ${ }^{4}$. A presença destas vulvovaginites é mais comum na faixa etária de 20 a 39 anos $^{5}$ e pode levar a complicações ginecológicas e obstétricas, como doença inflamatória pélvica, celulite pós-histerectomia, endometrite pós-aborto, corioaminionite e trabalho de parto prematuro ${ }^{6-10}$. Por outro lado, o processo inflamatório da mucosa vaginal induzido pela Candida spp. e pela Trichomonas vaginalis e a redução de lactobacilos que ocorre na VB facilitam a transmissão e/ ou infecção pelo HIV, além de aumentarem a replicação local deste vírus, favorecendo, assim, à penetração do vírus na mucosa ${ }^{11-14}$.

Ainda não está claramente estabelecido se existe uma maior freqüência de vulvovaginites, particularmente VB, em mulheres infectadas pelo HIV. Alguns estudos sugerem uma maior prevalência de vulvovaginites, como $\mathrm{VB}$, candidíase vaginal e tricomoníase, em mulheres infectadas pelo HIV ${ }^{15-18}$. Entretanto, o estudo da coorte Women's Interagency HIV Study (WIHS) não confirmou este achado; embora tenha encontrado uma elevada prevalência de VB em mulheres infectadas pelo HIV, a maior freqüência foi descrita em mulheres não infectadas ( 42 versus $48 \% ; \mathrm{p}=0,03)^{19}$. No Brasil, apenas uma coorte de mulheres infectadas pelo HIV-1 seguida no Rio de Janeiro abordou esta questão. As freqüências de VB, candidíase e tricomoníase nesta coorte foram de 18,6, 23,5 e 7\%, respectivamente ${ }^{20}$.

O presente estudo teve como objetivo avaliar a freqüência de vulvovaginites em mulheres infectadas pelo HIV e comparar com mulheres não infectadas. Além disso, foi avaliada a influência do uso de terapia antiretroviral e dos níveis de linfócitos T CD4 sobre a freqüência de vulvovaginites em mulheres infectadas pelo HIV.

\section{Métodos}

Trata-se de um estudo de corte transversal realizado no período de maio de 2006 a maio de 2007. O tamanho da amostra foi calculado considerando a prevalência de infecções vaginais em mulheres infectadas e não infectadas pelo HIV de 30 e $15 \%$, respectivamente, com poder estatístico de 0,08 e alfa 0.05. A amostra foi estimada em 108 mulheres para cada grupo. Entretanto, no decorrer do estudo foi observada uma diferença estatística significante entre os dois grupos de $25 \%$ e o tamanho da amostra foi recalculado para 61 pacientes por grupo, permanecendo o mesmo poder estatístico e alfa. Assim, foram incluídas 64 mulheres infectadas pelo HIV e 76 não infectadas (Grupo Controle), acompanhadas no Centro de Referência à Aids e no Ambulatório de Ginecologia da Escola Bahiana de Medicina e Saúde Pública da Fundação Bahiana para Desenvolvimento das Ciências (EBMSP/ FBDC), respectivamente. As pacientes foram incluídas seqüencialmente no momento que compareciam à consulta. Os critérios de inclusão foram: pacientes com idade superior a 18 anos, vida sexual ativa, sorologia positiva para HIV (Grupo HIV) ou negativa (Grupo Controle) e assinar o termo de consentimento livre e esclarecido. Foram excluídas mulheres no período gestacional ou puerperal, que utilizaram antibioticoterapia ou antifúngicos nos últimos 30 dias, e portadoras de doenças crônicas, como diabetes mellitus, lúpus eritematoso sistêmico, insuficiência renal crônica, ou em uso de corticosteróide. O estudo foi aprovado pelo Comitê de Ética em Pesquisa. As características da população estudada estão descritas na Tabela 1.

As mulheres infectadas pelo HIV tinham menor renda e escolaridade, comparadas às mulheres não infectadas. Além disso, naquele grupo, a vida sexual foi iniciada mais precocemente e o número de parceiros sexuais e a ocorrência de doenças sexualmente transmissíveis (DST) prévia foram significativamente superiores $(\mathrm{p}=0,004$; $\mathrm{p}<0,01$, respectivamente) - embora $73 \%$ delas referissem a utilização do condom (Tabela 1).

Todas as pacientes responderam a um questionário padrão, contendo dados demográficos, sintomas e sinais referidos e antecedentes ginecológicos e obstétricos e, em seguida, foram submetidas a exame ginecológico e coleta de material vaginal e de sangue periférico. $\mathrm{O}$ exame ginecológico, a coleta do material e o diagnóstico de VB foram realizados por dois ginecologistas nos ambulatórios de referência. Inicialmente, era feita a coleta de secreção vaginal com um swab para ser encaminhada à cultura, seguida de medida do $\mathrm{pH}$, coleta de secreção vaginal para realizar o teste das aminas, exame a fresco e Gram. Todas as amostras de sangue foram testadas para 


\begin{tabular}{|c|c|c|c|c|c|}
\hline & \multicolumn{2}{|c|}{$\begin{array}{l}\text { Sorologia negativa para HIV } \\
\qquad \mathrm{n}=76\end{array}$} & \multicolumn{2}{|c|}{$\begin{array}{l}\text { Sorologia positiva para HIV } \\
n=64\end{array}$} & \multirow{2}{*}{$\mathbf{p}$} \\
\hline & n & $\%$ & n & $\%$ & \\
\hline Idade (média, dp) & 76 & $28,6 \pm 5,9$ & 64 & $30,4 \pm 5,5$ & 0,07 \\
\hline União estável & 47 & 61,8 & 34 & 53,1 & 0,29 \\
\hline Tabagismo & 6 & 7,9 & 8 & 12,5 & 0,36 \\
\hline Anos de estudo (média, dp) & 76 & $9,1 \pm 3,1$ & 64 & $7,1 \pm 3,4$ & 0,001 \\
\hline \multicolumn{6}{|l|}{ Antecedentes sexuais e obstétricos } \\
\hline Idade $1^{a}$ RS (média, dp) & 76 & $17,5 \pm 3,9$ & 64 & $16,2 \pm 4,0$ & 0,05 \\
\hline Número de parceiros (média, dp) & 76 & $3,6 \pm 4,3$ & 64 & $8,3 \pm 13,4$ & 0,004 \\
\hline Uso atual de condom & 14 & 18,4 & 47 & 73,4 & $<0,01$ \\
\hline Antecedente de DST & 10 & 13,2 & 26 & 40,6 & $<0,01$ \\
\hline
\end{tabular}

$d p=$ desvio padrão; DST=doenças sexualmente transmissíveis; RS=relação sexual. $p<0,05$, teste do $\chi^{2}$ de Pearson, teste $t$ de Student, teste de Mann-Whitney.

HIV-1/2 por Enzyme-Linked ImmunoSorbent Assay (ELISA) e confirmadas por Western blot. A proporção de linfócitos T CD4+ e a carga viral foram obtidos dos registros dos prontuários. Foram selecionados apenas os resultados de exames realizados nos últimos seis meses anteriores à data da coleta.

A coleta da secreção vaginal foi realizada com swab umedecido em salina estéril. Após coleta, as amostras e os esfregaços da secreção foram encaminhados ao laboratório de microbiologia da EBMSP/FBDC. No laboratório, as amostras foram assepticamente semeadas no meio Sabouraud e incubadas a $35^{\circ} \mathrm{C}$ durante 24 a 48 horas para análise do crescimento de Candida spp. Os esfregaços da secreção foram corados pelo método de Gram e observados em microscópio. A leitura dos resultados foi realizada por duas microbiologistas, as quais não eram informadas sobre o estado sorológico da paciente quanto ao HIV.

Para o diagnóstico de VB, foram utilizados os critérios de Amsel, que considera positivo para tal infecção a presença de três dos quatro achados: corrimento vaginal, $\mathrm{pH}$ vaginal acima de 4,5 , teste das aminas positivo e presença de clue cells na avaliação microscópica ${ }^{21}$.

O diagnóstico de Trichomonas vaginalis foi feito pelo exame microscópico a fresco, imediatamente após a coleta do material vaginal.

As seguintes variáveis preditoras foram analisadas: idade (faixa etária), renda familiar, estado civil, tabagismo, anos de estudo, coitarca, número de parceiros sexuais, uso de condom, ocorrência de DST prévia, leucorréia, número de abortos, número de partos, dor pélvica, dor ao toque vaginal, infecção pelo HIV, uso de terapia antiretroviral, proporção de linfócitos $\mathrm{T}$ CD4 e carga viral.

As variáveis de desfecho foram definidas quanto à presença de VB, candidíase vaginal e Trichomonas vaginalis.
O teste do $\chi^{2}$ foi utilizado para comparação das variáveis categóricas, e, na sua impossibilidade, o teste exato de Fischer foi utilizado. O teste $t$ de Student foi empregado para estabelecer a significância estatística da diferença entre as médias dos grupos e, quando inadequado, o teste de Mann-Whitney. Foi realizada a análise de regressão logística múltipla para verificar a independência das associações. As variáveis ajustadas foram: infecção pelo HIV, estado civil, anos de estudo, renda familiar, tabagismo, passado de DST, uso de condom, número de parceiros sexuais e idade da primeira relação sexual. Foi adotado um nível de significância de 5\%. Para análise dos dados, foi utilizado o programa Statistical Package for Social Sciences (SPSS) 11.0.

\section{Resultados}

A infecção vaginal foi mais prevalente em pacientes infectadas pelo HIV (59,4\%), comparadas ao Grupo Controle $(28,9 \% ; \mathrm{p}<0,001)$, conforme Tabela 2 . Além disso, a $O d d s$ Ratio (OR) para infecção vaginal em mulheres infectadas pelo HIV foi de 2,7 (IC95\%=1,335,83; $\mathrm{p}=0,007)$, após ajuste das variáveis. As variáveis "renda familiar", "número de parceiros", "idade da primeira relação sexual”, "uso atual de condom”, "número de partos", "número de abortos" e "passado de DST" não foram isoladamente associada a uma maior prevalência de infecções vaginais. Escolaridade foi inversamente associado à prevalência das infecções vaginais $(\mathrm{OR}=0,29, \mathrm{IC} 95 \%=0,14-0,62)$.

Como observado na Tabela 2, a ocorrência de VB $(26,6 \%, p=0,04)$, candidíase vaginal $(29,7 \%, p=0,02)$ e tricomoníase $(12,5 \%, \mathrm{p}=0,04)$, isoladamente, foi maior no grupo de mulheres infectadas pelo HIV. Além disso, estas apresentavam maior freqüência de co-infecções $(9,4$ versus $1,3 \%, \mathrm{p}=0,05)$. 
Tabela 2 - Prevalência das infecções vaginais

\begin{tabular}{|c|c|c|c|c|c|}
\hline & \multicolumn{2}{|c|}{$\begin{array}{l}\text { Sorologia negativa para HIV } \\
n=76\end{array}$} & \multicolumn{2}{|c|}{$\begin{array}{l}\text { Sorologia positiva para HIV } \\
n=64\end{array}$} & \multirow{2}{*}{$\mathbf{p}$} \\
\hline & n & $\%$ & $n$ & $\%$ & \\
\hline Infecções vaginais & 22 & 28,9 & 38 & 59,4 & $<0,001$ \\
\hline Vaginose bacteriana & 10 & 13,2 & 17 & 26,6 & 0,04 \\
\hline Candidiase vaginal & 11 & 14,5 & 19 & 29,7 & 0,02 \\
\hline Tricomoníase & 2 & 2,6 & 8 & 12,5 & 0,04 \\
\hline
\end{tabular}

Co-infecção: associação entre duas ou mais infecções.

$p<0,05$; teste do $\chi^{2}$ de Pearson; teste exato de Fisher.

Tabela 3 - Características específicas da população infectada por HIV

\begin{tabular}{|c|c|c|c|c|c|}
\hline & \multicolumn{2}{|c|}{$\begin{array}{c}\text { Uso de TARV } \\
\quad \mathrm{n}=38\end{array}$} & \multicolumn{2}{|c|}{$\begin{array}{c}\text { Sem TARV } \\
n=26\end{array}$} & \multirow{2}{*}{$\mathbf{p}$} \\
\hline & n & $\%$ & n & $\%$ & \\
\hline Proporção de linfócitos T CD4 (média, dp) & \multicolumn{2}{|c|}{$579 \pm 556$} & \multicolumn{2}{|c|}{$738 \pm 439$} & 0,2 \\
\hline Carga viral $<1.000$ & 30 & $78,9 \%$ & 11 & $42,3 \%$ & 0,003 \\
\hline Infecção vaginal & 20 & $52,6 \%$ & 17 & $65,4 \%$ & 0,2 \\
\hline
\end{tabular}

TARV: terapia antiretroviral; Linfócitos TCD4: células $/ \mathrm{mm}^{3}$; Carga viral: cópias $/ \mathrm{mm}^{3}$.

$\mathrm{p}<0,05$; teste do $\chi^{2}$ de Pearson; teste de Mann-Whitney.

Tabela 4 - Frequiência das infecções vaginais de acordo com os níveis de linfócitos T CD4+

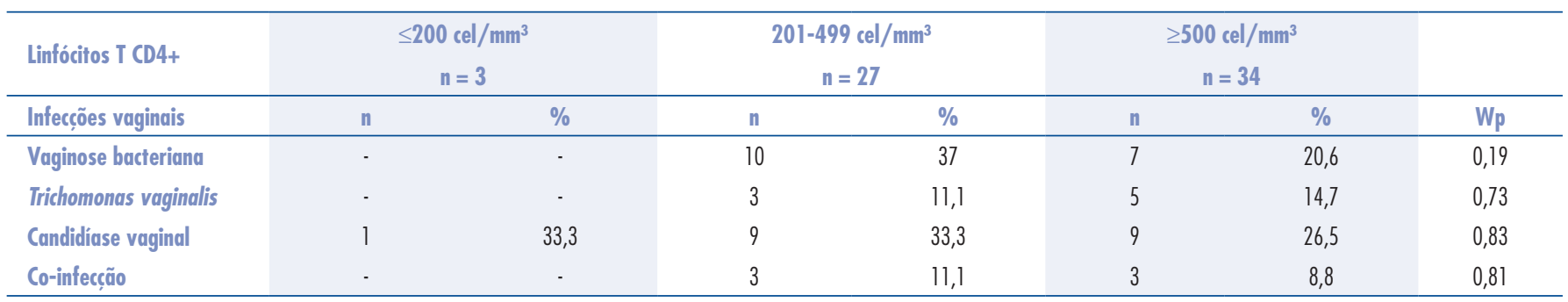

$\mathrm{p}<0,05$; teste do $\chi^{2}$ de Pearson; teste exato de Fisher.

Quanto à apresentação clínica das infecções vaginais no grupo de mulheres infectadas e não infectadas pelo HIV, a freqüência de fluxo vaginal e de prurido nas mulheres com VB, candidíase vaginal e tricomoníase foi semelhante entre os grupos.

Dentre as pacientes infectadas pelo HIV, 38 $(59,4 \%)$ utilizavam terapia antiretroviral. A média de linfócitos T CD 4 + foi de $644 \pm 514$ células $/ \mathrm{mm}^{3}$ e a média da carga viral foi de $7997 \pm 18338$ cópias/ $\mu \Lambda$. A proporção de linfócitos T CD4 foi semelhante entre pacientes que utilizavam ou não terapia antiretroviral; entretanto, a carga viral foi maior no grupo de mulheres que não utilizavam terapia antiretroviral. Não houve diferença na freqüência de infecção vaginal quando a amostra de mulheres infectadas pelo HIV foi subdividida de acordo com a utilização ou não de terapia antiretroviral (Tabela 3).

Quando analisamos apenas o grupo de mulheres infectadas pelo HIV, não observamos associação entre a freqüência de $\mathrm{VB}$, candidíase e tricomoníase e a proporção de linfócitos T CD4 (Tabela 4).

\section{Discussão}

Os resultados deste estudo indicam que a prevalência de infecções vaginais como VB, candidíase vaginal e tricomoníase é maior em mulheres infectadas pelo HIV. A candidíase vaginal e a tricomoníase são reconhecidamente mais prevalentes em mulheres infectadas pelo HIV. A candidíase vaginal é 3,8 vezes mais freqüente na presença de infecção pelo $\mathrm{HIV}^{18}$ e a prevalência de tricomoníase em mulheres infectadas pelo HIV varia de 9,5 a $38 \%{ }^{22}$, enquanto em mulheres não infectadas esta é observada em 1,4 a 4,5\% $\%^{18,23,24}$. Entretanto, a associação entre VB e infecção pelo HIV ainda não está completamente estabelecida. Uma maior freqüência de VB em mulheres infectadas pelo HIV tem sido relatada em alguns estudos ${ }^{15,18,25}$. O estudo HERS (Heart and Estrogen/Progestin Replacement Study) evidenciou que as mulheres imunocomprometidas apresentavam maior prevalência e persistência de VB, além de um quadro clínico mais grave, quando comparadas àquelas soronegativas ${ }^{25}$. Helfgott e al. ${ }^{18}$, avaliando uma amostra de 
159 mulheres infectadas pelo HIV e um grupo de 144 mulheres não infectadas, encontraram uma prevalência de VB de 25,6\% no grupo de mulheres infectadas pelo HIV e de $11 \%$ naquelas não infectadas. Contudo, a coorte WIHS descreveu uma maior prevalência de VB em mulheres não infectadas pelo HIV, comparadas a um grupo infectado ( 42 versus $48 \% ; \mathrm{p}=0,03)^{19}$. As variações nas proporções de VB poderiam ser parcialmente explicadas pelos critérios utilizados para o diagnóstico desta vulvovaginite e pelas características clínicas das pacientes envolvidas nos estudos. O diagnóstico de VB na coorte WIHS baseou-se no escore de Nugent et al. ${ }^{26}$, enquanto em nosso estudo e no de Helfgott e al. ${ }^{18}$, foram utilizados os critérios de $\mathrm{Amsel}^{21}$. Além disso, na coorte WIHS, não foram excluídas pacientes que utilizaram antibioticoterapia prévia, o que poderia explicar os resultados divergentes. Recentemente, no Brasil, um estudo avaliou uma coorte de 458 mulheres infectadas pelo HIV seguida no Rio de Janeiro. As prevalências de VB, candidíase e tricomoníase foram de 18,6, 23,5 e 7\%, respectivamente ${ }^{20}$. Porém, não houve um grupo controle de mulheres não infectadas. Estas freqüências foram inferiores às observadas em nosso estudo em Salvador, Bahia. Entretanto, a totalidade das pacientes da coorte do Rio de Janeiro utilizava terapia antiretroviral, enquanto $56 \%$ das mulheres do nosso estudo eram tratadas. Além disso, para diagnóstico de VB na coorte do Rio de Janeiro, foi utilizado o sistema de Nugent et al. ${ }^{26}$, que apresenta maior sensibilidade que os critérios de $\mathrm{Amsel}^{27}$.

Uma associação entre HIV e infecções vaginais poderia ser explicada por alterações imunes na mucosa vaginal, que podem ocorrer na presença do vírus.
Postula-se que a infecção pelo HIV reduz o número de linfócitos T CD4 + na mucosa vaginal ${ }^{18}$. Além disso, a VB em mulheres infectadas pelo HIV pode estar associada a concentrações elevadas de quimiocinas na mucosa vaginal ${ }^{28}$. Por outro lado, as infecções vaginais aumentariam a susceptibilidade à infecção pelo HIV, devido a uma menor produção de peróxido de hidrogênio por lactobacilos e à ruptura da barreira epitelial normal, favorecendo a infecção e replicação do vírus ${ }^{13}$.

Diante destes dados, é possível concluir que as infecções vaginais, principalmente a VB, a candidíase e a tricomoníase, são mais freqüentes em mulheres infectadas pelo HIV, independente do uso de condom. Assim, estas pacientes devem ser submetidas sistematicamente a avaliações ginecológicas cuidadosas e ao rastreamento das infecções vaginais.

\section{Agradecimentos}

Os autores agradecem a Doutora Margarida Santos Matos, chefe da disciplina de Ginecologia da EBMSP; Doutora Fabiana Bahia de Souza, que disponibilizou o ambulatório de Ginecologia do Centro de Referência de Aids (Creaids); Doutora Maria Lícia de Jesus dos Santos; Doutora Ana Gabriela Travassos e Doutora Virgínia Silva Lago, pelo acompanhamento das pacientes infectadas pelo HIV; Doutora Lúcia Castro, pela realização dos exames citopatológicos e os estudantes de Medicina, André Alves e Elaine Cravo, pelo processamento das amostras no Laboratório de Microbiologia. O projeto foi financiado pela Fundação de Amparo à Pesquisa do Estado da Bahia (Fapesb).

\section{Referências}

1. World Health Organization. UNAIDS. Progress on global access to HIV antiretroviral therapy: a report on " 3 by 5 " and beyond [report on the Internet]. Geneva: WHO/UNAIDS; 2006 [cited 2007 Jul 12]. Available from: http://www.who.int/hiv/progreport2006_en.pdf

2. Boletim Epidemiológico AIDST. Brasília, DF: Ministério da Saúde; 2004;1(1).

3. Wang J. Bacterial vaginosis. Prim Care Update Ob Gyns. 2000;7(5):181-5.

4. Adad SJ, de Lima RV, Sawan ZT, Silva ML, de Souza MA, Saldanha $\mathrm{JC}$, et al. Frequency of Trichomonas vaginalis, Candida sp and Gardnerella vaginalis in cervical-vaginal smears in four different decades. Sao Paulo Med J. 2001;1 19(6):200-5.

5. Fang $X$, Zhou $Y$, Yang $Y$, Diao $Y$, Li H. Prevalence and risk factors of trichomoniasis, bacterial vaginosis, and candidiasis for married women of child-bearing age in rural Shandong. Jpn J Infect Dis. 2007;60(5):257-61.
6. Hitti J, Hillier SL, Agnew KJ, Krohn MA, Reisner DP, Eschenbach DA. Vaginal indicators of amniotic fluid infection in preterm labor. Obstet Gynecol. 2001 ;97(2):21 1-9.

7. Genc MR, Witkin SS, Delaney ML, Paraskevas LR, Tuomala RE, Norwitz ER, et al. A disproportionate increase in IL-1 beta over IL- 1 ra in the cervicovaginal secretions of pregnant women with altered vaginal microflora correlates with preterm birth. Am J Obstet Gynecol. 2004; 190(5): $1191-7$.

8. Simoes JA, Hashemi FB, Aroutcheva AA, Heimler I, Spear GT, Shott $S$, et al. Human immunodeficiency virus type 1 stimulatory activity by Gardnerella vaginalis: relationship to biotypes and other pathogenic characteristics. J Infect Dis. $2001 ; 184(1): 22-7$

9. Platz-Christensen JJ, Sundström E, Larsson PG. Bacterial vaginosis and cervical intraepithelial neoplasia. Acta Obstet Gynecol Scand. $1994 ; 73(7): 586-8$. 
10. Sweet RL. Gynecologic conditions and bacterial vaginosis: implications for the non-pregnant patient. Infect Dis Obstet Gynecol. 2000;8(3-4): 184-90.

11. Cohen MS, Hoffman IF, Royce RA, Kazembe P, Dyer JR, Daly CC, et al. Reduction of concentration of HIV-1 in semen after treatment of urethritis: implications for prevention of sexual transmission of HIV-1. AIDSCAP Malawi Research Group. Lancet. 1997;349(9069):1868-73.

12. Laga M, Manoka A, Kivuvu M, Malele B, Tuliza M, Nzila N, et al. Non-ulcerative sexually transmitted diseases as risk factors for HIV-1 transmission in women: results from a cohort study. AIDS. 1993;7(1):95-102.

13. Sebitloane MH. HIV and gynaecological infections. Best Pract Res Clin Obstet Gynaecol. 2005;19(2):231-41.

14. Coleman JS, Hitti J, Bukusi EA, Mwachari C, Muliro A, Nguti R, et al. Infectious correlates of HIV-1 shedding in the female upper and lower genital tracts. AIDS. 2007;21(6):755-9.

15. Sewankambo N, Gray RH, Wawer M, Paxton L, McNaim $D$, Wabwire-Mangen $F$, et al. HIV-1 infection associated with abnormal vaginal flora morphology and bacterial vaginosis. Lancet. 1997;350(9077):546-50.

16. Rhoads JL, Wright DC, Redfield RR, Burke DS. Chronic vaginal candidiasis in women with human immunodeficiency virus infection. JAMA. 1987;257(22):3105-7.

17. Sorvillo F, Kovacs A, Kerndt P, Stek A, Muderspach L, SanchezKeeland $\mathrm{L}$. Risk factors for trichomoniasis among women with human immunodeficiency virus (HIV) infection at a public clinic in Los Angeles County, California: implications for HIV prevention. Am J Trop Med Hyg. 1998;58(4):495-500.

18. Helfgott A, Eriksen N, Bundrick CM, Lorimor R, Van Eckhout B. Vaginal infections in human immunodeficiency virus-infected women. Am J Obstet Gynecol. 2000; 183(2):347-55.

19. Greenblatt RM, Bacchetti P, Barkan S, Augenbraun M, Silver S, Delapenha $R$, et al. Lower genital tract infections among HIV-infected and high-risk uninfected women: findings of the Women's Interagency HIV Study (WIHS). Sex Transm Dis. 1999;26(3):143-51.
20. Grinsztejn B, Bastos Fl, Veloso VG, Friedman RK, Pilotto JH, Schechter $M$, et al. Assessing sexually transmitted infections in a cohort of women living with HIV/AIDS, in Rio de Janeiro, Brazil. Int J STD AIDS. 2006; 17(7):473-8.

21. Amsel R, Totten PA, Spiegel CA, Chen KC, Eschenbach D, Holmes KK. Nonspecific vaginitis. Diagnostic criteria and microbial and epidemiologic associations. Am J Med. $1983 ; 74(1): 14-22$.

22. DeHovitz J, Kelly P, Feldman J, Sierra MF, Clarke L, Bromberg $J$, et al. Sexually transmitted diseases, sexual behavior, and cocaine use in inner-city women. Am J Epidemiol. 1994; 140(12): 1 125-34.

23. Sutton MY, Sternberg M, Nsuami M, Behets F, Nelson AM, St Louis $M E$. Trichomoniasis in pregnant human immunodeficiency virusinfected and human immunodeficiency virus-uninfected congolese women: prevalence, risk factors, and association with low birth weight. Am J Obstet Gynecol. 1999; 181 (3):656-62

24. Van Der Pol B, Kwok C, Pierre-Louis B, Rinaldi A, Salata RA, $\mathrm{Chen} \mathrm{PL}$, et al. Trichomonas vaginalis infection and human immunodeficiency virus acquisition in African women. J Infect Dis. 2008; 197(4):548-54.

25. Jamieson DJ, Duerr A, Klein RS, Paramsothy P, Brown W, Cu-Uvin $S$, et al. Longitudinal analysis of bacterial vaginosis: findings from the HIV epidemiology research study. Obstet Gynecol. $2001 ; 98(4): 656-63$

26. Nugent RP, Krohn MA, Hillier SL. Reliability of diagnosing bacterial vaginosis is improved by a standardized method of gram stain interpretation. J Clin Microbiol. 1991;29(2):297-301.

27. Moussavi Z, Behrouzi R. Diagnostic Amsel criteria compared standardized method of Gram stain for the diagnosis of bacterial vaginosis. Int Congr Ser. 2004; 1271:392-5.

28. Losikoff P, Fichorova R, Snyder B, Rodriguez I, Cu-Uvin S, Harwell $J$, et al. Genital tract interleukin-8 but not interleukin-1 beta or interleukin-6 concentration is associated with bacterial vaginosis and its clearance in HIV-infected and HIV-uninfected women. Infect Dis Obstet Gynecol. 2007;2007: 92307. 